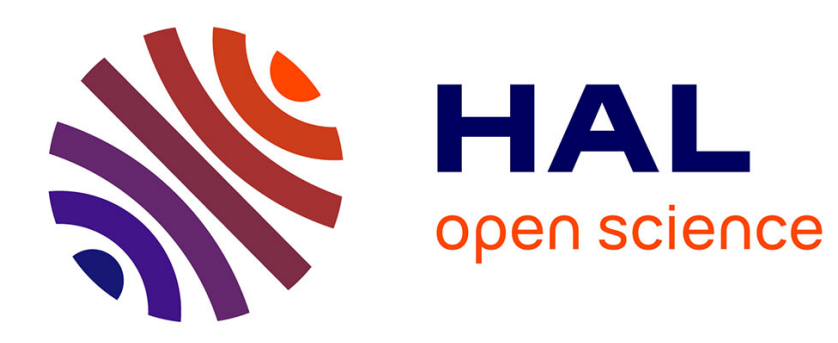

\title{
LA LITTÉRATURE POUR PENSER L'ÉCOLOGIE POSTCOLONIALE CARIBÉENNE
}

\author{
Malcom Ferdinand
}

\section{To cite this version:}

Malcom Ferdinand. LA LITTÉRATURE POUR PENSER L'ÉCOLOGIE POSTCOLONIALE CARIBÉENNE. Multitudes, 2015, 10.3917/mult.060.0065 . halshs-02289758

\section{HAL Id: halshs-02289758 \\ https://shs.hal.science/halshs-02289758}

Submitted on 17 Sep 2019

HAL is a multi-disciplinary open access archive for the deposit and dissemination of scientific research documents, whether they are published or not. The documents may come from teaching and research institutions in France or abroad, or from public or private research centers.
L'archive ouverte pluridisciplinaire HAL, est destinée au dépôt et à la diffusion de documents scientifiques de niveau recherche, publiés ou non, émanant des établissements d'enseignement et de recherche français ou étrangers, des laboratoires publics ou privés. 


\title{
LA LITTÉRATURE POUR PENSER L'ÉCOLOGIE POSTCOLONIALE CARIBÉENNE
}

\author{
Malcom Ferdinand
}

Association Multitudes | « Multitudes »

2015/3 n $60 \mid$ pages 65 à 71

ISSN 0292-0107

Article disponible en ligne à l'adresse :

https://www.cairn.inforevue-multitudes-2015-3-page-65.htm

Distribution électronique Cairn.info pour Association Multitudes.

(C) Association Multitudes. Tous droits réservés pour tous pays.

\section{(cc) BY-NC}




\section{La littérature \\ pour penser l'écologie postcoloniale caribéenne \\ Malcom Ferdinand}

Les colonisations européennes des Amériques et de l'archipel caribéen dès la fin du XVe siècle ont entraîné des changements sans commune mesure dans cette région du monde. Outre sa violence manifeste à travers les conquêtes coloniales, les traites négrières et les esclavages, cette rencontre entre l'ancien monde et le nouveau monde engendra aussi de profonds changements écologiques. Si les modes coloniaux d'exploitation des terres portèrent atteinte à la biodiversité et diminuèrent les ressources naturelles, ces changements écologiques concernèrent également des modes de vie, des conceptions de la terre, des pratiques, des cultures et des récits créant une véritable "révolution écologique coloniale ${ }^{1}$ ». En effet, l'écologie ne peut se réduire à la seule étude des changements environnementaux. Le sens donné à la terre, à la nature aux paysages ou à l'écologie se manifeste à travers un ensemble de pratiques sociales et culturelles, de récits ordinaires et mythiques, d'actions politiques et de formes d'organisation économique. L'écologie qui en tant que science étudie les «rapports des organismes avec le monde extérieur» tel que l'a formulé Ernst Haeckel en $1866^{2}$, ne peut se penser indépendamment des hommes et de leurs diverses activités. Ne serait-ce que par leurs activités de production, les hommes coconstruisent cette nature et cette terre, participant pleinement à l'écologie d'une région. Plus encore, à travers leurs pratiques sociales et culturelles, leurs organisations économiques et politiques, les différentes sociétés créent des récits à partir desquels la nature, la terre et l'écologie prennent sens. La nature et l'environnement demeurent ainsi des éléments constitutifs d'un imaginaire social comprenant des conceptions du monde et de son entour, des manières de vivre-ensemble entre humains et non humains. Une telle acception de l'écologie comprenant

1 Merchant, Carolyn, Ecological revolutions: Nature, Gender, and Science in New England, The University of North Carolina Press, Chapel Hill \& Londres, 1989.

2 Cité par Deléage, Jean-Paul, Une histoire de l'écologie, La Découverte, Paris, 1991, p. 63. 


\section{Multitudes 60}

ses relations physiques, biologiques, mais aussi narratives et imaginaires avec les sociétés, nous pousse à dépasser la vision environnementaliste moderne qui réduit la terre et la nature à des objets totalement extérieurs et séparés des hommes, fussent-ils à préserver. Penser l'écologie caribéenne nous amène donc non seulement à nous interroger sur les changements physiques, les modifications chimiques et biologiques survenues, mais aussi à explorer les récits et imaginaires à travers lesquels apparaissent ces terres et ces mers.

\section{Une Caraïbe sans récit?}

Or les spécificités historiques de la Caraïbe posent une double difficulté à cette recherche. En effet, les colonisations et esclavages européens qui ont fondé ces sociétés caribéennes ont posé comme une impossibilité de ce que l'on peut nommer ici des récits écologiques. Nous connaissons aujourd'hui le bilan «humain» des colonisations européennes de la Caraïbe. Par leurs conquêtes armées et par cet «échange colombien ${ }^{3}$ » que représente la propagation de maladies européennes sur ces terres, ces colonisations eurent pour première conséquence la disparition presque totale des populations amérindiennes présentes avant l'arrivée de Christophe Colomb. Pour exemple, la population des Taïnos de l'île d'Hispaniola - actuellement partagée par Haïti et Saint-Domingue - passa d'un nombre estimé à plus d'un million en 1492 à environ 500 en $1535^{4}$. Cependant, au-delà de cette disparition physique de peuples entiers, au-delà de la violence inouïe des conquêtes et mises en esclavage, ces colonisations entraînèrent des pertes qui ne peuvent être exprimées par l'arithmétique du nombre de morts, d'espèces disparues ou encore d'arbres coupés. Avec cette disparition rapide, s'éteignirent également un ensemble de récits, de pratiques culturales et religieuses par lesquels ces habitants donnaient sens à ces îles et à leur présence sur celles-ci. Cette extinction littérale et littéraire de l'indigénat explique en partie la faible présence de revendications de protection de l'environnement qui à l'instar des récits du continent américain ferait appel à une cosmologie ancestrale et sacrée. Cette disparition pour Édouard Glissant «a déraciné le sacré ${ }^{5} »$. Contrairement à la Pachamama de Bolivie et autres cosmogonies des peuples autochtones de l'Amérique de la cordillère andine aux extrémités nord du Canada en passant par les Native Americans des États-Unis, ces îles caribéennes font face à une impossibilité de ce sacré du fait des modalités "génocidaires» des colonisations européennes. De ce fait, les îles de l'archipel caribéen ne furent plus une Terre-mère. Le génocide des Amérindiens fut accompagné du matricide de cet archipel. Ces enfants de la Terre ayant disparu, la relation par laquelle une mère prend soin de ses enfants et en retour ceux-là vénèrent celle-ci en en prenant soin fut rompue. Cette rupture créa, reprenant le phrasé du créole martiniquais, une «Terre-sans-manman». Il s'agit d'une terre perdue, une terre sans attache qui, démise de sa dignité de nourrice, ne ferait plus l'objet de souci.

3 Crosby, Alfred, The Columbian exchange: biological and cultural consequences of 1492, Greenwood Publishing, Westport, 1973.

4 D’Ans, André-Marcel, Haïti: paysage et société, Khartala, Paris, 1987, p. 51-61.

5 Glissant, Édouard, Poétique de la Relation, Gallimard, Paris, 1990, p. 161. 
Parallèlement à cet effacement des récits écologiques précolombiens, le développement du commerce triangulaire dans ces îles comprenant une économie de plantation dans un régime esclavagiste et la traite négrière transatlantique donnèrent lieu à une autre difficulté. L'arrachement de millions d'Africains de leurs lieux de vie, de leurs pratiques et de leurs activités artistiques, suivi de leur enfermement dans les entrailles des navires négriers avant de travailler dans les plantations engendra ce que le poète Robert Nesta Marley nomma un «bottomless pit» (un trou sans fond) ${ }^{6}$. Édouard Glissant symbolise cette rupture par la figure d'un gouffre où presque tout se perd ${ }^{7}$. Pour Glissant ce gouffre se déploie dans trois dimensions: le gouffre du ventre du navire négrier, la cale refermée où furent jetés nombre d'Africains captifs, le gouffre d'une navigation sans repère au milieu d'un océan dont l'absence de limites perçues en fait une mer infinie, et enfin le gouffre de l'abandon de pays, de communautés, de terres et d'être chers que l'on ne reverra jamais. Se perdirent de la sorte des cultures, des langues, des images et des récits. Ainsi, outre les millions de déplacés, les innommables souffrances de la traite négrière, la violence singulière de peuples amérindiens entièrement décimés, les équilibres écologiques écroulés et le rythme sanglant d'un esclavage multiséculaire, peut-être que l'une des conséquences les plus importantes des colonisations européennes dans la Caraïbe demeure l'apparente disparition de récits. Avec la mort de ces voix amérindiennes, avec ces chaînes enlacées à ces chevilles africaines, disparaissent des chants, des danses, des pratiques, des croyances et surtout des récits.

Si Philippe Descola ${ }^{8}$ nous enseigne que le «Grand Partage» entre Nature et Culture caractéristique de la modernité est une construction relativement récente de l'occident, relativement minoritaire au regard des autres sociétés du monde et de leurs figures du continu, peu de régions du monde connurent une telle manifestation de ce partage. Des terres furent littéralement séparées de leurs habitants tandis que d'autres - Européens, Africains et Asiatiques - furent amenés de gré ou de force donnant cet assemblage baroque que constituent les sociétés créoles aujourd'hui. Dans une telle situation, quels imaginaires, quels récits seront mobilisés pour penser la terre, plus encore l'écologie aujourd'hui?

\section{Une écologie coloniale}

Commençons par remarquer que la réponse à cette recherche ne se trouve pas du côté d'une écologie strictement scientifique. Rappelons que dans le prolongement de la double utopie coloniale d'une terra nullius et d'une nature vierge', les premières politiques écologiques dans cette région maintinrent cette séparation entre terres et habitants de ces terres. Les premières expériences de conservation de forêt se déroulèrent dès le XVIII ${ }^{e}$ siècle au sein des

6 Marley Robert Nesta, «Redemption Song » in Bob Marley \& the wailers, Uprising, Island records, 1980.

7 Glissant, Édouard, Poétique de la relation, Gallimard, Paris, 1990, p 18.

8 Descola, Philippe, Par-delà nature et culture, Gallimard, Paris, 2005.

9 Denevan, William, «Le mythe de la nature vierge. Le paysage des Amériques en 1492 », in Hache, Emilie (dir.), Écologie politique, cosmos, communauté, milieux, Amsterdam, Paris, 2012, p. 283-316. 
anciennes colonies européennes de l'Océan Indien (l'̂̂le Maurice) et des Caraïbes tels que Saint-Vincent, Trinidad et Tobago. Pierre Poivre accompagné de Bernardin de Saint-Pierre et de Philibert Commerson mit en place des politiques et des expériences de conservation de forêts jouant un rôle fondamental dans le développement de ce courant scientifique. Si selon l'historien Richard Grove, ces expériences contribuèrent «à une réflexion analytique rigoureuse sur les processus de changement écologique et à la formation d'une idéologie de conservation ${ }^{10}$ ", elles demeurèrent en revanche totalement étrangères aux esclaves d'origines africaines et aux Amérindiens habitant ces terres à l'époque. Elles révélaient ainsi une manière de penser la préservation de l'environnement ne tenant pas compte des cultes et des pratiques de ces forêts par ceux-là même qui y demeuraient. L'exclusion de la majeure partie de la population de ces projets de conservation ne réside pas dans l'emploi de main-d'œuvre et d'aide locale pour leur mise en place, mais se situe plutôt sur les plans sémantiques et ontologiques. Par cette opération, soudainement un espace, un biotope à un temps donné se retrouve sectionné des liens physiques, culturels, politiques mais surtout narratifs par lesquels il participe au monde: l'écologie est privée de ses récits. Non seulement la forêt est pensée indépendamment de ceux qui l'ont habitée et vénérée, mais elle devient par la même détachée des récits et histoires de ces derniers qui jadis l'habitaient en fruits invisibles.

Deux siècles plus tard, cette pensée particulière de l'écologie coloniale a perduré par endroits. Certains discours de type "écologie profonde» eurent tendance à prolonger cette séparation des hommes et de l'environnement ${ }^{11}$. Dans cette conception d'une planète sans homme, «la nature», «la terre», «l'environnement» sont érigés en objets dont les définitions ne comprennent pas d'autres modalités de relation avec les hommes que celle de l'impact - et le plus souvent l'impact négatif. Les hommes dégradent l'environnement. Ils sont donc exclus de celui-ci quand paradoxalement par leurs protections, par leurs associations écologistes, par leurs politiques publiques, par leurs partis politiques écologistes et par leurs conférences internationales, ils n'ont peut-être jamais été dans des relations aussi intimes avec cette «nature».

À de multiples reprises lors de mes rencontres avec des associations environnementalistes de Guyane, de Porto Rico, de Guadeloupe ou d'Haïti, des membres déplorent que le fait que les locaux «ne s'intéressent pas ou peu à l'environnement». Certaines associations internationales viendraient alors en secours à cette terre-sans-manman pour laquelle personne a priori ne parle. Ce constat rejoint l'idée que les pays pauvres, de par leur urgence économique et leur pauvreté chronique, ne voudraient pas ou tout simplement ne seraient pas en mesure de prendre soin de leur environnement et de leur nature. À la question «qui parle quand l'environnement et/ou la nature entre en jeu? », la faim qui resserre le ventre bâillonnerait également

10 Grove Richard, Les îles du Paradis. L'invention de l'écologie aux colonies.1660-1854, La Découverte, Paris, 2013 (Futurs antérieurs), p. 18-19.

11 Voir Frazier Nash Roderick, Wilderness and the American mind, Yale University Press, New Haven et Londres, 2001, p. 383. 
ceux et celles qui localement tenteraient d'agir et de narrer. Par cet "environnementalisme des riches ${ }^{12} »$ déjà critiqué par Ramachandra Guha et Juan Martinez-Allier, les pauvres sont dépourvus d'initiatives écologistes ou de pratiques environnementales alternatives. Réduisant l'écologie à un environnementalisme, cette pensée et ses pratiques, malgré «les bonnes intentions», renforcent l'ontologie coloniale du monde caribéen en maintenant cette séparation entre ces espaces et ceux qui y vivent. Face à cette histoire politique et sociale qui tend à occulter des récits et face à une conception de l'écologie faisant fi des récits, l'écologie dans cette région serait-elle à jamais marquée par le vide narratif de cette ontologie coloniale? Quelle possibilité existe-t-il pour une autre écologie dans ces espaces?

\section{La littérature pour penser l'écologie postcoloniale caribéenne}

La littérature offre des outils pour penser l'écologie qui me semblent particulièrement pertinents pour la Caraïbe. Depuis une vingtaine d'années un courant d'étude littéraire, l'écocriticism, interroge les relations entre les hommes et leur environnement à partir de ces récits oraux et écrits. Il s'agit, déclare l'une des fondateurs de ce courant Cheryll Glotfelty, d'étudier les relations entre «la littérature et l'environnement physique ${ }^{13} »$ :

«[si] nous acceptons la première loi de l'écologie posée par Barry Commoner postulant que "tout est relié à tout le reste", nous devons conclure que la littérature ne flotte pas au-dessus du monde matériel dans une sorte d'éther esthétique, mais au contraire, joue un rôle dans un système mondial extrêmement complexe, dans lequel l'énergie, la matière et les idées interagissent ${ }^{14}$.»

En ce sens, la littérature pourrait nous permettre de saisir ces entrelacements complexes et ces récits liant ensemble les hommes et de leurs environnements dans les Caraïbes tant dans leurs relations matérielles qu'immatérielles. Si les colonisations et esclavages dans les Caraïbes ont tu des histoires, des cosmogonies, des dieux et des récits, tout n'a pas été perdu. Restent des traces. Des traces de résistance à ce transbordement, à ces esclavages et à ces extinctions littéraires. Des traces de cris dans ce navire négrier marquant pour Raphaël Confiant et Chamoiseau la naissance de la littérature créole. Des traces de danses, de croyances, de chants, et de récits. Des traces dont les combinaisons créatrices et le souvenir inventif participent du phénomène de créolisation ${ }^{15}$. Ces traces guettées et suivies par les écrivains et poètes caribéens qui en véritables archéologues de l'imaginaire donnent dans leurs écrits d'inspirations écologistes à nouveau naissance à ces récits qui mettent en scène les relations entre humains et non-humains.

12 Guha, Ramachandra \&, Martinez-Allier, Juan, «L'environnementalisme des riches», in Hache, Emilie (dir.), Écologie politique, cosmos, communauté, milieux, Amsterdam, Paris, 2012, p. 51-65.

13 Republié dans Glotfelty, Cheryll \& Fromm, Harold (ed), The ecocriticism reader: landmarks in literary ecology, University of Georgia Press, Londres, 1996.

14 Ibid., p. XIX.

15 Glissant, Edouard, Introduction à une poétique du divers, Gallimard, Paris, 1996. 


\section{Multitudes 60}

Nous pouvons y trouver par exemple des relations politiques. L'un des premiers à avoir réussi à penser ensemble l'exigence de préservation des ressources naturelles avec les luttes pour l'égalité et la liberté d'un peuple est l'écrivain haïtien Jacques Roumain. Dans son roman Gouverneurs de la rosée $e^{16}$ paru en 1944, des habitants ruraux dominés politiquement par les autorités de la ville se retrouvent dans une situation de misère en partie due à la sécheresse des mornes. En articulant la recherche de l'eau et la préservation des ressources naturelles à leur lutte pour l'égalité, ces habitants entrevoient une possible émancipation. À travers ce roman, Romain en précurseur raconte déjà comment la situation écologique d'Haïti demeure intimement liée à la sortie de la pauvreté chronique des habitants et l'émancipation des formes d'assujettissement postcoloniales. Nous y trouvons aussi la quête d'une mémoire de l'histoire portée par les paysages de la terre et de la mer. Le poète et dramaturge saint-lucien Derek Walcott plonge dans les profondeurs de la mer à la recherche de ces traces et récits:

"Where are your monuments, your battles, martyrs?

Where is your tribal memory? Sirs,

in that grey vault. The sea. The sea

has locked them up. The sea is History ${ }^{17}$ »

Les terres, les mers et les rivières deviennent des lieux de mémoires d'une histoire terrible. Le poète de Guyane anglaise Martin Carter s'agenouille et écoute la Terre pensant entendre les voix des esclaves morts dans son poème Listening to the land (Écoutant la terre). L'écrivaine Edwige Danticat, dans son recueil d'histoires Krik? Krak! ${ }^{18}$, raconte de quelle manière l'eau se révèle grosse d'une mémoire douloureuse à travers les multiples évadées haïtiennes qui finissent dans les profondeurs de la mer au large de la Floride ou encore cette rivière qui devint rouge sang lors du massacre d'Haïtiens à la frontière de la République Dominicaine.

Encore, certains poètes par leurs écritures retranscrivent les paroles de la terre, des pierres et des animaux se transformant en véritables « appareils de phonation ${ }^{19}$ ». La poète américaine Maya Angelou dans son poème A rock, A river, a Tree engage un dialogue entre un rocher, une rivière et un arbre d'une terre américaine et les hommes de diverses origines qui y ont séjourné. Cette archéologie est aussi explicite dans la démarche scripturale de Patrick Chamoiseau par laquelle, au-delà de ces écrits écologistes, il procède à une mise en parole de ces traces. Comme cet ossement affleurant découvert en plein bois au pied d'une pierre gravée par des Caraïbes à partir duquel il imagine et raconte L'esclave vieil homme et le molosse ${ }^{20}$, comme cette ombre furtive surplombante à partir de laquelle il raconte Les neuf consciences $d u$ malfini ${ }^{21}$, l'on ne sait plus si c'est l'écrivain ou si ce sont ces traces qui parlent.

16 Roumain, Jacques, Gouverneurs de la rosée, Haïti: Imprimerie de l'État, Port-au-Prince, 1944.

17 Walcott, Derek, "The Sea is history», in Selected Poems, New York, Straus et Giroux, 2008, traduction par l'auteur «Où sont vos monuments, vos batailles, vos martyrs?/ Où est votre mémoire tribale? Messieurs,/ dans ce caveau gris.

La mer. La mer/ les a tous enfermés. La mer est l'Histoire».

18 Danticat, Edwige, krik? Krak!, Soho, New York, 1995.

19 Latour, Bruno, politiques de la nature: comment faire entrer les sciences en démocratie, La Découverte, Paris, 1999.

20 Chamoiseau, Patrick, L'esclave vieil homme et le molosse, Gallimard, Paris, 1997.

21 Chamoiseau, Patrick, Les Neufs consciences du malfini, Gallimard, Paris, 2009. 
Si cette recherche littéraire concernant ces écologies postcoloniales s'est développée au cours de ces dix dernières années à travers le courant de l'écocritique postcoloniale ${ }^{22}$, cette recherche des récits écologiques ne concerne pas uniquement les écrivains. Des associations écologistes locales créent aussi leurs récits, à travers leurs écrits mais aussi leurs actions politiques. C’est le cas par exemple de l'association martiniquaise nommée ASSAUPAMAR (Association pour la Sauvegarde du Patrimoine Martiniquais). Créée à la fin des années 1970, cette association a mené de nombreuses manifestations et actions en justice à l'encontre de projets menaçant les écosystèmes de la Martinique. La reconnaissance de l'importance de ces récits écologiques motiva une rencontre entre membres de cette association et écrivains donnant lieu au recueil de textes Écrire pour la Terre, Écrire pour l'ASSAUPAMAR ${ }^{23}$. Dans ce recueil, dix-huit écrivains et intellectuels ont écrit des contes, des poèmes, des nouvelles qui mettent en scène des histoires et des trames mêlées à la terre, sa faune, sa flore et à ses habitants. Loin d'instaurer cette alternative infertile entre la terre et les hommes, ces narrations montrent la portée d'un dire de la terre:

«Nous attendons en effet, ce roman où notre Terre ne sera pas objet d'une relation aussi fertile soit-elle mais sujet du dire ${ }^{24}$.»

Ces divers récits constituent autant de voix qui font de la terre non plus un objet, mais bien un sujet parmi d'autres sujets. Une terre qui n'est plus esseulée, pensée sans tous ceux dont les pieds la caressent, mais bien avec ceux qui l'habitent.

Cette recherche des récits écologiques dans la Caraïbe et cette importance du dire ne constituent pas un ensemble d'ornements qui viendrait illustrer la «vraie» tâche des écologistes, celle consistant à préserver la biodiversité, les ressources naturelles ou prévenir les pollutions environnementales. Ces récits demeurent au contraire la texture narrative et imaginaire même des actions écologiques. L'écologie n'est pas a-historique et encore moins sans récit. À travers les préoccupations de préservation de la planète, de la biodiversité et des ressources, des récits et des histoires sont à écrire! Ces narrations écologistes écrites ou orales aujourd'hui dans les Caraïbes, région du monde où les récits furent tus par les colonisations et esclavages, comportent alors une portée décoloniale en ce qu'elles manifestent des formes d'associations et des liens entre les habitants, humains et non-humains, et leurs milieux. Elles font émerger des mémoires cachées derrière ces arbres et ces montagnes, ces rivières et ces champs de cannes. La mise en paroles de ces multiples non-humains, ce dire à travers ces littératures, témoigne de la nécessité de penser l'émancipation postcoloniale des Caribéens avec leurs relations à leurs paysages, leurs terres et leurs écologies. Ces récits deviennent des praxis décoloniales par lesquelles les militants et écrivains manifestent non seulement leur engagement dans la préservation des écosystèmes de ces terres avec un souci pour les générations futures, mais montrent aussi, comme l'affirme Aimé Césaire, la présence déterminée de «ceux sans qui la terre, ne serait pas la terre ${ }^{25}$ ».

\footnotetext{
22 Voir par exemple, Deloughrey, E., Gosson, R. \& Handley, G., Caribbean literature and the environment: between nature and culture, University of Virginia press, Charlotesville, 2005.

23 ASSAUPAMAR (dir.), Écrire pour la Terre, Écrire pour l'ASSAUPAMAR, Éditions ASSAUPAMAR, Lamentin Martinique, 1989.

24 Ibid., p. 4.

25 Césaire, Aimé, Cahier d’un retour au pays natal, Présence Africaine, Paris, 1983 (1 1 ère édition, Revue Volonté 1939), p. 44.
} 